\title{
Long-Distance Interval Training Following Pre-Cooling With an Ice Vest
}

Ronald L. Hager

ron_hager@byu.edu

lain Hunter

iain_hunter@byu.edu

Gary W. Mack

Andrew R. Tegeder

Follow this and additional works at: https://scholarsarchive.byu.edu/facpub

Part of the Exercise Science Commons

\section{Original Publication Citation}

Tegeder A, Hunter I, Mack G, \& Hilton S. (28). "Long-Distance Interval Training Following PreCooling with an Ice Vest". International Journal of Sports Science and Coaching, 3(2), 269-275.

\section{BYU ScholarsArchive Citation}

Hager, Ronald L.; Hunter, lain; Mack, Gary W.; and Tegeder, Andrew R., "Long-Distance Interval Training Following Pre-Cooling With an Ice Vest" (2008). Faculty Publications. 910.

https://scholarsarchive.byu.edu/facpub/910 


\section{Long-Distance Interval Training Following Pre-Cooling With an lce Vest}

\section{Andrew R. Tegeder, lain Hunter, Gary W. Mack and} Ron Hager

Department of Exercise Sciences, Brigham Young University, 120D Richard's Building, Provo, UT 84602

Email: iain_hunter@byu.edu

\section{ABSTRACT}

This study evaluated how preparing for an interval workout, while wearing a cooling vest, affects core temperature $\left(T_{c}\right)$, heart rate $(H R)$, and interval time in long-distance runners. Nineteen male collegiate cross-country runners exercised under two different conditions: (a) wearing a Nike PreCoolTM ice vest for one hour prior to the workout (30 minutes resting with the vest and 30 minutes while performing their warm-up); and (b) a control condition utilizing a traditional warm-up. Subjects performed $8 \times$ $1000 \mathrm{~m}$ intervals. HR and T were measured prior to warm-up, immediately before the first interval, and after each interval. $T_{c}$ measured before the first interval was significantly lower in the vest condition (difference $=0.37^{\circ} \pm$ $0.2^{\circ} \mathrm{C}$ ). Differences persisted through the sixth interval. Differences in $\mathrm{HR}$ and interval times were nonsignificant between conditions for all intervals. Wearing an ice vest prior to and during warm-up effectively lowers $T_{c}$ during long-distance interval training.

Key words: Body Temperature, Exercise, Performance, Precooling, Running

\section{INTRODUCTION}

One of the limits to endurance exercise is internal body core temperature $\left(\mathrm{T}_{\mathrm{c}}\right)$. When $\mathrm{T}$ reaches a critical limiting level of $\sim 39^{\circ} \mathrm{C}$ to $40^{\circ} \mathrm{C}$, the individual may experience a reduction in exercise performance $[1,2]$. In theory, if endurance athletes had the ability to prolong the time to reach this critical $T_{c}$ then there may be the possibility of limiting the decline in exercise performance resulting from a high endogenous heat load. One method to achieve a reduced $\mathrm{T}_{c}$ is to precool the body.

Reducing $\mathrm{T}_{c}$ prior to exercise improves running performance [3-5]. Various methods to precool subjects have been used such as immersion in cold water, exposure to cold air, wearing a liquid-perfused garment or wearing a cooling vest $[3,4,6,7]$. Several companies have designed vests that are proposed to reduce $T_{c}$ in athletes before competition. These cooling vests hold frozen ice packs in close contact with the body. Because of their relatively light weight, athletes are able to wear vests during the warm-up directly prior to an event One recent study showed that $T_{c}$ (rectal temperature), skin temperature, and esophageal temperature all decreased during warm-up with a cooling vest [3]. More importantly, time to

Reviewer: $\quad$ Rob Duffield (Charles Sturt University, Australia) 
complete a 5-km simulated race was reduced following a warm-up with a cooling vest, which was associated with a reduction in heart rate (HR) when athletes exercised under precooled conditions [3]. This leads to speculation that athletes may be able to perform more work if they precool before competition or training. Previous studies evaluating cooling vests have focused on the effects the vest might have on performance during a simulated race or longduration, submaximal exercise. This study will evaluate the use of a cooling vest as a training aid for long-distance runners. Long-distance runners routinely perform interval workouts during training. Other precooling studies have only looked at the effect precooling has on continuous exercise. Therefore, the aim of this study was to investigate the effect of wearing a cooling vest during warm-up on $\mathrm{T}_{c}$, HR and interval session time during interval training in NCAA Division I distance runners in an outdoor setting.

\section{METHODS}

SUBJECTS

Nineteen healthy subjects were recruited from a NCAA Division I men's cross-country team. Subjects were homogenous in age (21.2 yrs $\pm 0.6 \mathrm{yrs})$, height $(1.81 \mathrm{~m} \pm 0.8 \mathrm{~m})$, mass $(68.1$ $\mathrm{kg} \pm 0.02 \mathrm{~kg})$, and body surface area $\left(1.87 \mathrm{~m}^{2} \pm 0.03 \mathrm{~m}^{2}\right)$. All subjects provided written informed consent and all procedures were approved by the institutional review board at Brigham Young University.

\section{TESTING PROTOCOL AND PROCEDURES}

Each subject warmed up and exercised under two different conditions: (a) an experimental condition in which subjects wore a cooling vest (Nike, Beaverton, OR) during warm-up; and (b) a control condition in which subjects wore a regular T-shirt during warm-up. The testing days were separated by 6 days, and groups were randomly assigned (randomized crossover design) which day they wore the cooling vest during warm-up. Each subject served as his own control. All subjects started each interval as a group in each of the two workouts.

Subjects reported for testing after a two-hour fast. However, subjects were allowed to drink as much water as they desired. Subjects were instructed not to consume caffeine 12 hours before testing. Three hours before reporting for testing, each subject swallowed a telemetry pill. This was sufficient time for the pill to enter the GI tract. Each subject's $T_{c}$ was recorded prior to putting on the cooling vest. Subjects put on the cooling vest 60 minutes prior to the workout. The first 30 minutes included passive rest with the vest on, followed by 30 min of warming up while still wearing the cooling vest. The warm-up protocol before the training sessions was the same on each day and consisted of 15 minutes of jogging, followed by stretching, and four $100 \mathrm{~m}$ strides at the workout pace. Before putting on the vest, subjects placed a heart-rate monitor around their chest. Subjects were allowed to drink as much water as they wanted during warm-up and in between intervals. This matches how athletes would typically ingest fluids during a workout rather than having consumption regulated for them.

After the warm-up, subjects removed the cooling vest and ran eight 1000-m intervals separated by 90 seconds. Each subject performed the intervals with a group of runners with similar running abilities. The coach gave each group a minimum time requirement in which they had to complete each interval during the training session. $\mathrm{HR}$ and $\mathrm{T}_{\mathrm{c}}$ were measured prior to warm-up, just prior to start of the first interval, and after each interval. Time to complete each interval was also recorded.

\section{TESTING EQUIPMENT}

$\mathrm{T}_{c}$ was measured using the CorTemp $\mathrm{T}_{c}$ monitoring system (HQ Inc., Palmetto, FL). The 
| Sports Science 3-2_final 30/6/08 2:30 pm Page 271

CorTemp system consists of a small precalibrated ingestible sensor (telemetry pill) which measures and transmits $\mathrm{T}_{c}$. Each subject swallowed a sensor (about the size of a large vitamin) three hours before $T_{c}$ was measured. $T_{c}$ was measured with a monitor after the pill entered the subject's GI tract. HR was measured by a Polar HR monitor (Polar Electro, Woodbury, NY) and reported in beats per minute (bpm). Environmental conditions at the workout site (dry bulb temperature, wet bulb globe temperature (WBGT), and relative humidity) were measured using a heat stress indicator (Microtherm Heat Stress W136T, Casella, Amhurst, NH) while wind speed was taken from measurements at a university weather station located within one mile of the testing site.

Interval times were recorded by research assistants with stopwatches. Heart rates were recorded by research assistants based on displayed values on the HR monitor's watch immediately after they crossed the finish line of each interval.

\section{STATISTICAL ANALYSIS}

Data were analyzed using a repeated-measures ANOVA to determine the significance of the means by condition (vest vs. non-vest) using the SAS System for Univariate and Multivariate Statistics (version 9.2). Level of significance was set at 0.05 and all data are represented as means \pm SE. Significant differences of $\mathrm{HR}, \mathrm{T}$ and interval times between conditions at any given measurement period were determined by computing a minimum significant difference (MSD) value using Tukey's honest significant difference method.

\section{RESULTS}

TEMPERATURES AND HUMIDITY ON TESTING DAYS

Average dry bulb temperature for the first day of testing was $24.6^{\circ} \mathrm{C} \pm 0.17^{\circ} \mathrm{C}$, relative humidity was $39.9 \% \pm 2.7 \%$, WBGT was $19.9^{\circ} \mathrm{C} \pm 0.15^{\circ} \mathrm{C}$, and wind speed was $1.15 \mathrm{~m} / \mathrm{s} \pm$ $0.01 \mathrm{~m} / \mathrm{s}$. Average dry bulb temperature on the second day of testing was $31.1^{\circ} \mathrm{C} \pm 0.07^{\circ} \mathrm{C}(\mathrm{p}$ $<0.05$, different from day 1), relative humidity was $38.9 \% \pm 0.3 \%$, WBGT was $24.7^{\circ} \mathrm{C} \pm$ $0.06^{\circ} \mathrm{C}(\mathrm{p}<0.001$, different from day 1$)$, and wind speed was $1.09 \mathrm{~m} / \mathrm{s} \pm 0.01 \mathrm{~m} / \mathrm{s}$.

$T_{C}$ RESPONSES: BASELINE, WARM-UP AND DURING 1000 M INTERVALS Repeated measures ANOVA observed a difference between vest and non vest groups $(\mathrm{p}<$ 0.001). Significant differences between groups for each $T_{c}$ measurement were determined using critical values of the studentized range. Thus, individual $p$-values were not computed for each comparison, but significance was determined by whether a difference at any point in time fell within the studentized range. Baseline $\mathrm{T}_{\mathrm{c}}$ measured prior to warm-up or precooling period were similar between conditions (vest $=37.1^{\circ} \mathrm{C} \pm 0.08^{\circ} \mathrm{C}$, non vest $=$ $37.24^{\circ} \mathrm{C} \pm 0.14^{\circ} \mathrm{C}$ ). $\mathrm{T}_{\mathrm{c}}$ measured directly prior to the first interval was significantly lower in the vest condition than the non-vest condition (vest $=37.05^{\circ} \mathrm{C} \pm 0.22^{\circ} \mathrm{C}$, non vest $=37.42^{\circ} \mathrm{C}$ $\pm 0.2^{\circ} \mathrm{C}$ ). Figure 1 shows average $\mathrm{T}_{\mathrm{c}}$ for each condition. This significant difference between $\mathrm{T}_{c}$ persisted through the end of the $6^{\text {th }}$ interval. After the $7^{\text {th }}$ and $8^{\text {th }}$ intervals, differences between conditions were not significant.

Plateaus for $\mathrm{T}$ in both conditions were found at similar values (vest $=38.78^{\circ} \mathrm{C}+0.02^{\circ} \mathrm{C}$; non-vest $=38.84^{\circ} \mathrm{C} \pm 0.02^{\circ} \mathrm{C}$ ). The vest group reached its peak $\mathrm{T}_{\mathrm{c}}$ after the $7^{\text {th }}$ interval and the non-vest group reached peak $\mathrm{T}_{\mathrm{c}}$ by the $5^{\text {th }}$ interval. The vest condition $\left(0.051^{\circ} \mathrm{C} / \mathrm{min} \pm\right.$ $\left.0.01^{\circ} \mathrm{C} / \mathrm{min}\right)$ had a slightly lower rate of $\mathrm{T}_{\mathrm{c}}$ increase $(\mathrm{p}=0.07)$ than the non-vest condition $\left(0.066^{\circ} \mathrm{C} / \mathrm{min} \pm 0.01^{\circ} \mathrm{C} / \mathrm{min}\right)$. 
HR AND INTERVAL TIME RESPONSES DURING $1000 \mathrm{~m}$ INTERVALS

No significant differences were observed in the HR response $(p=0.94$, Fig. 2$)$ or interval times between the vest and non-vest conditions $(p=0.81$, Fig. 3 ).

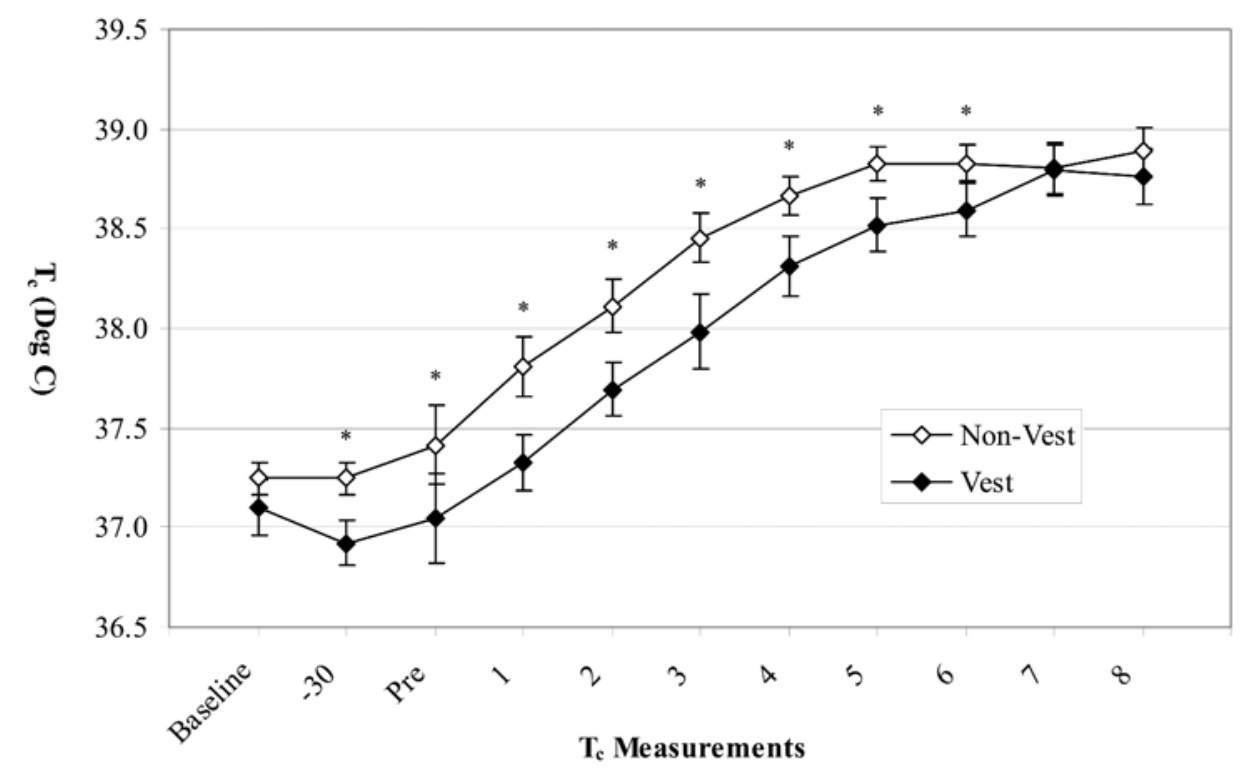

Figure 1. Average Body Core Temperatures $\left({ }^{\circ} \mathrm{C}\right)$ Measured at Baseline, Prior to Warm-Up Period, Just Before the 1st Interval, and Immediately Following Each $1000 \mathrm{~m}$ Interval

Values are mean \pm SE.

* denotes significantly different from Vest condition $(p<0.05)$

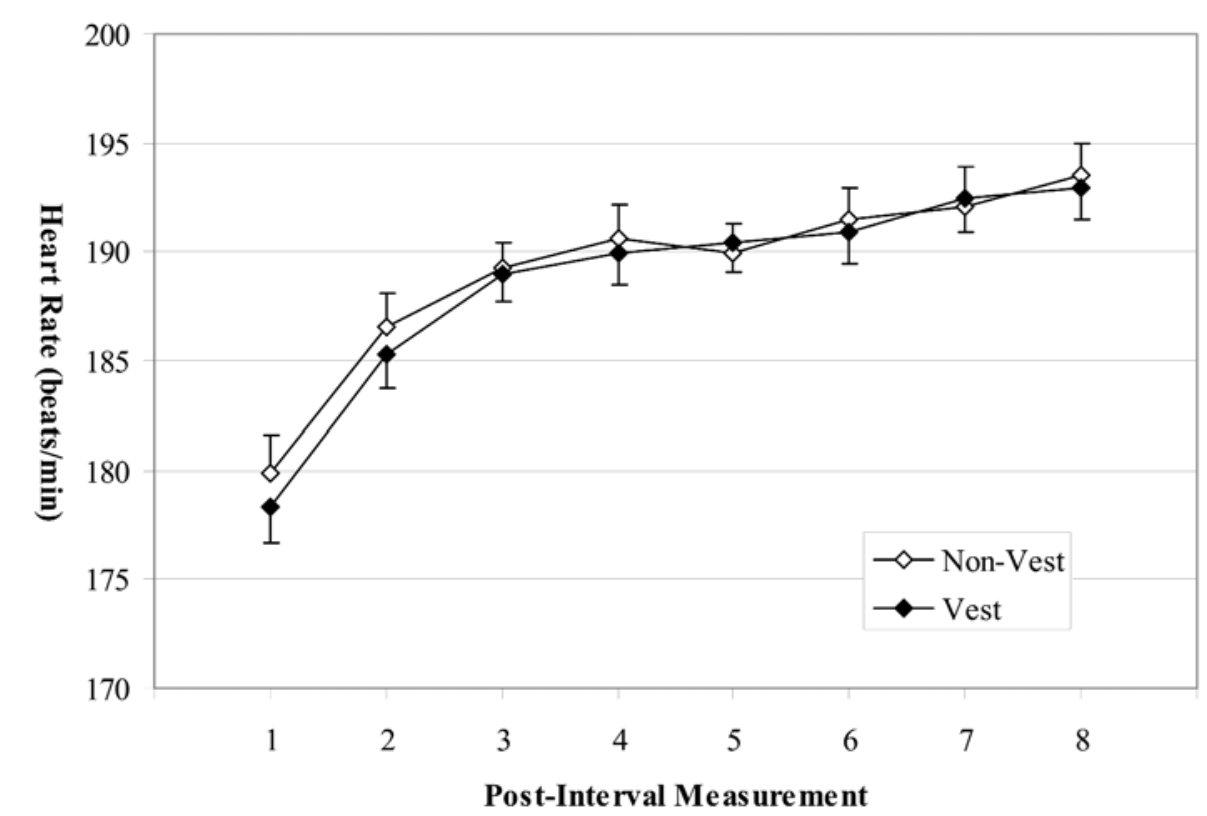

Figure 2. Average Heart Rates (bpm) Measured Directly After Each 1000 m Interval

Values are mean \pm SE. 


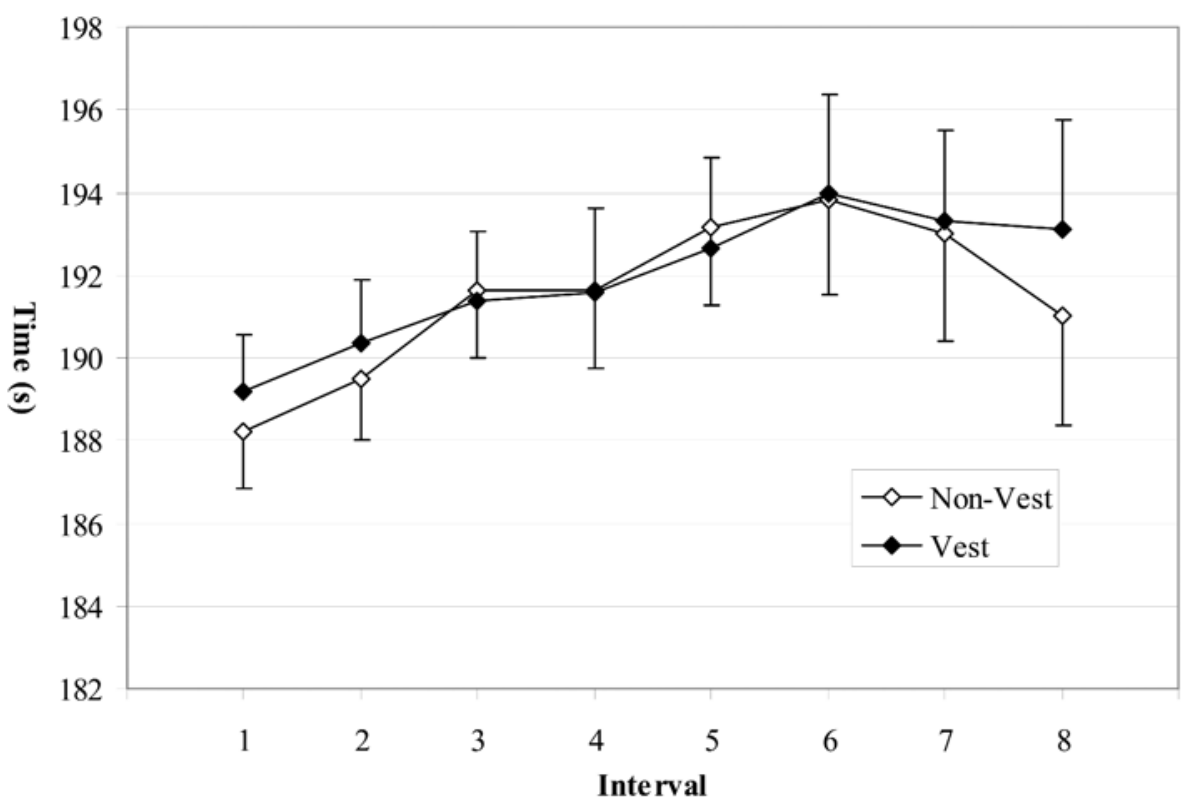

Figure 3. Average Time (s) to Complete Each 1000 m Interval Values are mean \pm SE.

\section{DISCUSSION}

The most important observation of this study is that precooling with an ice vest reduced $T_{c}$ and delayed the time to reach the peak $\mathrm{T}$ of $38.8^{\circ} \mathrm{C}$. As such, the subjects trained at a lower $\mathrm{T}_{\mathrm{c}}$ for approximately 30 minutes of an interval workout. Despite the reduction in $\mathrm{T}_{\mathrm{c}}$, there was no marked impact on the average interval time or HR.

The reduction in $\mathrm{T}$ observed was similar to those found in previous precooling studies employing a cooling vest $[3,8]$. The magnitude of the decrease in $\mathrm{T}_{\mathrm{c}}$ following precooling is dependent on the technique used. For example, whole-body cooling using either water immersion or cold air exposure has resulted in a substantially larger decrease in $\mathrm{T}_{c}$ than precooling with an ice vest $[2,8,9]$. The unique aspect to precooling with an ice vest is that it is more practical to athletes, because it can be used while performing their typical warmup activities. Relatively few studies have employed cooling vests as a means of precooling. One study using a cooling vest found that precooled subjects had reduced 5-km run times [3, 9]. Our study demonstrated a similar reduction in $T_{c}$ during the warm-up period prior to an interval workout, but no change in interval split-time.

The results of our study confirm the ability of upper-body cooling with an ice vest to significantly reduce $T_{c}$ during exercise in a warm environment. However, no performance benefit was observed during this high intensity, intermittent exercise workout. This result is in contrast to previous studies that have reported an improvement in exercise performance after precooling [1, 4, 8, 10-12]. White et al. [7] reported that both whole-body precooling and lower-body precooling reduced $\mathrm{T}_{\mathrm{c}}$. More importantly, lower-body precooling reduced metabolic demand and further reduced the perceptual effect associated with whole-body precooling. Cotter et al. [6] compared cycling performance between three groups: a control group, an ice vest group, and an ice vest plus thigh cooling group. They found that both precooled groups performed better than the control group during continuous exercise in the 
heat, but there was no difference between the two precooled groups [9]. Their results suggest that an ice vest alone provides a benefit to performance without the need to specifically cool other parts of the body or those muscles directly involved with the exercise. The difference in mode, duration, and pacing strategy of exercise in our study may explain lack of improvement in performance measures that were seen in other long duration tests.

The unique aspect of this study is the use of high-intensity long-distance interval running as the exercise mode. One previous study by Drust et al. [13] focused on the effects of precooling on $\mathrm{T}_{c}, \mathrm{HR}$, and other physiological responses to soccer-specific intermittent exercise. The exercise protocol consisted of subjects running on a treadmill at varying speeds for specific periods of time. The protocol consisted of static pauses, walking, jogging, submaximal high-intensity running, and sprinting. Subjects in this study ran on a treadmill, simulating the type of exercise intensities they encountered in soccer. The researchers did not report significant differences for oxygen consumption, HR, or rectal temperature between conditions. They concluded that precooling may have limited benefit for soccer-specific intermittent exercise. It is possible that the improvement in exercise performance with a reduction in $\mathrm{Tc}$ is not manifest in a high-intensity, intermittent exercise setting.

Results of this study show that during the interval workout, $\mathrm{T}_{\mathrm{c}}$ reached a plateau of $38.8^{\circ} \mathrm{C}$ $\pm 0.3^{\circ} \mathrm{C}$ in both groups. The rate of increase in $\mathrm{T}$ tended to be lower $(P=0.07)$ in the vest group $\left(0.056^{\circ} \mathrm{C} / \mathrm{min} \pm 0.01^{\circ} \mathrm{C} / \mathrm{min}\right)$ than the non-vest group $\left(0.064^{\circ} \mathrm{C} / \mathrm{min} \pm 0.01^{\circ} \mathrm{C} / \mathrm{min}\right)$. The rate of heat storage is dependent on the balance between heat production, related to metabolic rate or training pace; and heat dissipation, related to radiant and convective heat loss and evaporative heat loss. The similar rates of heat storage during the interval workouts reflect the similar rates of metabolic heat production and similar balance of heat dissipating mechanisms. The cooling vest allowed for the athletes to reduce the initial $\mathrm{T}_{c}$ during the warm-up period and thereby store more heat during the interval workout. The plateau in $T_{c}$, the point at which the rate of heat production and heat dissipation were equal and therefore the rate of heat storage was equal to zero, occurred earlier in the non-vest group. A higher capacity to store heat should mean less thermal strain during exercise in conditions where heat dissipating mechanisms are unable to match metabolic heat production. While the improved heat dissipation did not improve interval performance in the present study, this increased heat-loss capacity may be of benefit in the current and hotter conditions for medical, health and comfort purposes.

The nature of this field study imposes specific limitations, including the environmental factors. Differences in dry bulb temperature and WBGT on the testing days may have limited our ability to compare these two sets of data. However, the plateau in $\mathrm{T}$ during each workout reached similar levels on both days (first testing day $=38.66^{\circ} \mathrm{C} \pm .07^{\circ} \mathrm{C}$; second day $=$ $38.82^{\circ} \mathrm{C} \pm .06^{\circ} \mathrm{C}$ ). Based on the work of Lind [14], both WBGT values fell below the upper limit of the expected prescriptive zone for our experimental conditions (WBGT and exercise intensity). As such, comparing the $\mathrm{T}_{\mathrm{c}}$ response for vest and non-vest conditions from these two days was deemed acceptable. In addition, repeated measures ANOVA did not reveal a time-by-treatment interaction for $T_{c}$, HR, or interval times. This may indicate that each of these variables responded similarly for each condition on the two testing days.

\section{CONCLUSION}

Precooling with an ice vest effectively lowered $T_{c}$ in trained athletes completing a longdistance interval workout. The lower $\mathrm{T}_{\mathrm{c}}$ was apparent through six out of eight $1000 \mathrm{~m}$ intervals. However, both interval time performance and HR were not affected by the precooling technique. It is proposed that precooling with an ice vest during the warm up may 
be beneficial to reduce the thermoregulatory load or improve the heat-loss capacity during exercise, which may be of particular benefit in warmer environments.

\section{ACKNOWLEDGMENT}

Thank you to Ira and Mary Lou Fulton for funding this study.

\section{CONFLICT OF INTEREST}

Nike provided this study with cooling vests, some body core temperature monitoring equipment, and an individual who assisted with data collection. To avoid any bias coming from the assistant from Nike, the individual only helped a small group of students with operating the temperature measurement device. The readings from the monitor were viewed and recorded by students working with the primary investigator. The results of the present study do not constitute endorsement of the product by the authors.

\section{REFERENCES}

González-Alonso, J., Teller, C., Anderson, S. L., Jensen, F. B., Tyldig, T. and Nielsen, B., Influence of Body Temperature on the Development of Fatigue During Prolonged Exercise in the Heat, Journal of Applied Physiology, 1999, 86, 1032-1039.

2. Olschewski, H. and Brück, K., Thermoregulatory, Cardiovascular, and Muscular Factors Related to Exercise After Precooling, Journal of Applied Physiology, 1988, 64, 803-811.

3. Arngrímsson, S. Á., Petitt, D. S., Stueck, M. G., Jorgensen, D. K. and Cureton, K. J., Cooling Vest Worn During Active Warm-Up Improves 5-km Run Performance in the Heat, Journal of Applied Physiology, 2004, 96, 1867-1874.

4. Booth, J., Marino, F. and Ward, J. J., Improved Running Performance in Hot Humid Conditions Following Whole Body Precooling, Medicine and Science in Sports and Exercise, 1997, 29(7), 943-949.

5. Brück, K. and Olschewski, H., Body Temperature Related Factors Diminish the Drive to Exercise, Canadian Journal of Physiology and Pharmacology, 1987, 65, 1274-1280.

6. Cotter, J. D., Sleivert, G. G., Roberts, W. S. and Febbraio, M. A., Effects of Pre-Cooling, With and Without Thigh Cooling, on Strain and Endurance Exercise Performance in the Heat, Comparative Biochemistry and Physiology, 2001, 128, 667-677.

7. White, A. T., Davis, S. L. and Wilson, T. E., Metabolic, Thermoregulatory, and Perceptual Responses During Exercise After Lower vs. Whole Body Precooling, Journal of Applied Physiology, 2003, 94, 1039-1044.

8. Lee, D. T. and Hayes, E. M., Exercise Duration and Thermoregulatory Responses after Whole Body Precooling, Journal of Applied Physiology, 1995, 79, 1971-1976.

9. Marino, F. E., Methods, Advantages, and Limitations of Body Cooling for Exercise Performance, British Journal of Sports Medicine, 2002, 36, 89-94.

10. Hessemer, V., Langush, D., Brück, K., Bödeker, R. H. and Breidenbach, T., Effect of Slightly Lowered Body Temperatures on Endurance Performance in Humans, Journal of Applied Physiology, 1984, 56, 1731-1737.

11. Kay, D., Taaffe, D. R. and Marino, F. E., Whole-Body Precooling and Heat Storage During Self-Paced Cycling Performance in Warm Humid Conditions, Journal of Sports Sciences, 1999, 17, 937-942.

12. Schmidt, V. and Brück, K., Effects of a Precooling Maneuver on Body Temperature and Exercise Performance, Journal of Applied Physiology, 1981, 50, 772-778.

13. Drust, B., Cable, N. T. and Reilly, T., Investigation of the Effects of the Pre-Cooling on the Physiological Responses to Soccer-Specific Intermittent Exercise, European Journal of Applied Physiology, 2000, 81, 1117.

14. Lind, A. R., A Physiological Criterion for Setting Thermal Environmental Limits for Everyday Work, Journal of Applied Physiology, 1963, 18, 51-56. 